\title{
Proposta para o desenvolvimento de taxonomias em portais corporativos
}

Luciane Paula Vital

\begin{abstract}
Mestre em Ciência da Informação. Professora do Departamento de Ciência da Informação da Universidade Federal de Pernambuco.
\end{abstract}

\section{Ligia Café}

Doutora em Linguística. Professora do
Departamento de Ciência da Informação da
Universidade Federal de Santa Catarina.

O trabalho pretende, respaldado teórica e metodologicamente pelas áreas da Classificação, Indexação, Linguística e Documentação, apresentar uma proposta de desenvolvimento de taxonomias. A taxonomia tem como proposição classificar e ordenar as informações em áreas específicas do conhecimento, como apoio à gestão, tanto da informação quanto do conhecimento. A pesquisa toma por base a análise de práticas de elaboração de taxonomia identificadas na literatura. Trata, na primeira parte, da construção da taxonomia, apresentando seu desenvolvimento pautado em cinco etapas. A segunda parte é dedicada à aplicação da taxonomia na representação da informação $e$ organização dos documentos recuperados. Apesar das recomendações objetivarem o desenvolvimento de taxonomias no ambiente corporativo, suas indicações podem ser aplicadas a qualquer ambiente, realizando-se as devidas adaptações. Podemos constatar que a taxonomia trabalha os conceitos básicos da classificação aplicados ao ambiente digital.

Palavras-chave: Taxonomia; Representação da informação; Organização da informação; Representação do conhecimento.

\section{Proposal for the development of taxonomies in enterprise portals}

The work aims, supported by theoretical and methodological areas of the Classification, Indexing, 
Linguistics and Documentation, submit a proposal to develop taxonomies. The taxonomy has the proposition of to classify and sort the information in specific areas of knowledge such as management support, of both information and knowledge. Is based on the analysis of elaboration practices of taxonomy identified in the literature. Deals, in the first part, with the construction of the taxonomy, presenting its development lined into five stages. The second part is devoted to the application of the taxonomy in the representation of information and organization of documents retrieved. Despite the recommendations having the goal of developing taxonomies in the corporate environment, their statements can be applied to any environment, making the necessary adaptations. We note that the taxonomy works the basic concepts of classification applied to the digital environment.

Keywords: Taxonomy; Information representation; Information Organization; Knowledge representation.

Recebido em 07.10.2011 Aceito em 12.12.2011

\section{Introdução}

A taxonomia, vista como um sistema de classificação, é definida por Langridge (1987, p. 25) como "um mapa completo de qualquer área do conhecimento, mostrando todos os seus conceitos e suas relações [...]", sendo um objeto de estudo antigo da Documentação. Porém, no contexto dos ambientes organizacionais, sua aplicação é relativamente recente. Nesse meio, ela surge como uma forma de melhorar a organização da informação em portais corporativos, possibilitando o tratamento adequado dos documentos, com o objetivo de recuperá-los.

Apesar de se respaldar em algumas etapas de desenvolvimento de tesauros, entendemos a taxonomia como um modelo diferenciado, que tem como proposição classificar e ordenar as informações em áreas específicas do conhecimento, como apoio à gestão, tanto da informação quanto do conhecimento. Para Martinez et al. (2004, p. 106), "a taxonomia, em um sentido amplo, é a criação da estrutura (ordem) e dos rótulos (nomes) que ajudam a localizar a informação relevante. Em um sentido mais específico, é o ordenamento e rotulação de metadados, que permite organizar sistematicamente a informação primária". Além disso, a sua aplicação é indicada na construção de portais, tendo como característica o uso dos recursos inerentes a esse ambiente.

Terra e Gordon (2002, p. 53) dizem que "os portais são fundamentais para organizações intensivas no uso de informação e conhecimentos, [assim como] para organizações que se comunicam e 
colaboram de forma intensiva com um número expressivo de agentes e atores externos (clientes, parceiros, fornecedores)". Segundo os autores, os portais melhoram a eficiência do trabalho em equipe, o uso de informações corporativas e do ambiente externo e podem aumentar a produtividade do trabalhador do conhecimento. Dessa maneira, enquanto os portais corporativos exercem a função de armazenamento e difusão da informação produzida pelas empresas, a taxonomia atua como instrumento mediador entre aquele que registra o conhecimento em forma de informação e o que busca e recebe a informação procurada. Nesse sentido, pode-se dizer que a taxonomia corporativa é um índice dos vários documentos dispersos na empresa, e contribui na obtenção de altos níveis de relevância no processo de recuperação. Assim, ela está estreitamente ligada às teorias e métodos elaborados pelas áreas de Classificação, Indexação, Linguística e Documentação, nos quais deve apoiar-se.

Respaldado teórica e metodologicamente por essas áreas, apresentamos uma proposta, tomando por base a análise de práticas de elaboração de taxonomia identificadas na literatura (EARLEY AND ASSOCIATES, 2003; DUTRA; BUSCH, 2003; KREMER; KOLBE; BRENNER, 2005; YAMAOKA, 2005; MONTAGUE INSTITUTE, 2001; MORANTE, 2003). O trabalho trata, na primeira parte, da construção da taxonomia, enquanto a segunda é dedicada à aplicação da taxonomia na representação da informação e organização dos documentos recuperados. Apesar das recomendações objetivarem o desenvolvimento de taxonomias no ambiente corporativo, suas indicações podem ser aplicadas a qualquer ambiente, realizando-se as devidas adaptações.

\section{Construção da taxonomia}

A construção da taxonomia corporativa refere-se ao processo de representação do conhecimento existente no ambiente corporativo. Seu desenvolvimento deve estar orientado pelas cinco etapas descritas a seguir.

\subsection{Estabelecimento das categorias gerais}

O estabelecimento das categorias, como primeira etapa de construção da taxonomia, deve seguir tanto uma abordagem dedutiva como indutiva.

Segundo Vickery (1960), no método dedutivo, estabelecem-se as categorias gerais em um trabalho de equipe, entre especialistas, profissionais da informação e usuários, de acordo com as necessidades percebidas por esse grupo. A esse grupo cabe prever todas as possíveis categorias que representem o universo informacional da organização, constituindo, assim, a estrutura geral da taxonomia.

A literatura considera a Teoria da Classificação Facetada, definida por Ranganathan como a principal representante deste método. Nela, são definidas as categorias fundamentais as quais demarcam a primeira classificação de assuntos dentro de um grande universo. Campos (2001, 
p. 53) reconhece que "são elas que fornecem a visão de conjunto dos agrupamentos que ocorrem na estrutura, possibilitando, assim, o entendimento global da área".

Ranganathan (1962) instituiu cinco categorias fundamentais:

a) personalidade - É considerada indefinível, quando um termo não se adapta a nenhuma outra categoria, é entendida como uma manifestação desta.

b) matéria - materiais constitutivos de um objeto. Ex: mesa/Matéria: madeira;

c) energia - processos, operações, ações, técnicas, métodos, fenômenos;

d) espaço - lugares; e

e) tempo - períodos.

Alguns autores apontam a insuficiência dessa lista e sugerem sua ampliação. É o caso, por exemplo, do elenco de categorias proposto pelo Classification Research Group (CRG) (1955), exposto abaixo.

\subsubsection{Tipos de produto final}

- partes - divisões do produto final;

- materiais - constituintes do produto final;

- propriedades - qualidades do produto final;

- processos - ações ou reações das próprias coisas;

- operações - ações sofridas pelas coisas;

- agentes - responsáveis pelas ações;

- espaço - lugar;

- tempo - época; e

- forma de apresentação - tipo de documento.

Segundo Piedade (1977, p. 13), nas categorias propostas pelo CRG, "a razão de ser do estudo do assunto a classificar é o seu produto final". No caso de um setor de desenvolvimento de sistemas, por exemplo, o produto final é o software; suas partes são os diferentes aplicativos; seu material é o disco óptico; a propriedade, como qualidades do produto final, é, no caso do software, a portabilidade; os processos são a instalação dos programas, o gerenciamento de dados; as operações são planejar, projetar e desenhar; o agente é o programador; e a forma de apresentação é o $c d$-rom, por exemplo.

Esses dois exemplos, trazidos da literatura da área de Biblioteconomia, podem servir como guia para o mapeamento das categorias existentes no ambiente corporativo. Observamos, no entanto, que, devido à natureza do objeto desse tipo de taxonomia, categorias que representam 'o que é produzido' (objeto), 'quem produz' (agente) e 'como é produzido' (processo) são imprescindíveis. Nesse sentido, o esquema proposto pelo CRG parece ser mais apropriado.

Juntamente com o método dedutivo, as categorias podem ser estabelecidas pela abordagem indutiva, que busca defini-las de acordo com as diferentes fontes da área. Vickery (1960) diz que essas categorias 
podem ser determinadas pela consulta à literatura especializada da área, como dicionários, tesauros, artigos, relatórios técnicos, etc. É importante que se tome como base classificações ou estruturas de informação existentes e se possa validá-las por um grupo de especialistas ou representantes das diferentes áreas da organização. Como orientação ao método indutivo, citamos as orientações apresentadas pelas práticas de elaboração de Earley and Associates (2003), Dutra e Busch (2003) e Kremer, Kolbe e Brenner (2005) que observam a importância de:

- questionar-se sobre que categorias são importantes para o grupo (trabalho cooperativo denominado 'autoria do conhecimento');

- pesquisar sobre as formas preferenciais de navegação e classificação em sites utilizadas pelo grupo; e

- conhecer as maneiras pessoais de organização de cada integrante do grupo.

As categorias gerais precisam denotar características fundamentais do objeto/processo para o qual a taxonomia está sendo desenvolvida. Observamos, finalmente, a necessidade de denominar corretamente as categorias, notando aspectos de variação conceitual e terminológica.

\subsection{Coleta dos termos}

Na compilação dos termos, a consulta à literatura especializada ou classificações existentes na organização deve sempre ser utilizada como recurso auxiliar. De acordo com Ramsden (1974 apud PIEDADE, 1977), como os termos precisam fazer sentido ao grupo de usuários que irá utilizá-los, a literatura especializada serve como validação dos termos selecionados. Sendo assim, dois princípios devem ser levados em consideração no momento da coleta de termos: a garantia literária e a garantia de uso.

Lancaster (1987, p. 21), ao tratar sobre o tema garantia literária, registra que "[...] um termo se justifica apenas se ocorre dentro da literatura de um determinado assunto com algum grau de freqüência". Esse princípio pode ser aplicado quando se coletam termos de fontes de referência significativas da área, que pode ser um tesauro ou publicações de resumos, por exemplo. Com os sistemas automatizados e as bases de dados, esse trabalho torna-se mais rápido e eficiente.

Quanto ao conceito de garantia de uso, os termos coletados precisam representar as necessidades dos usuários. Lancaster (1987, p. 25) afirma que "alguns termos podem ocorrer na literatura, mas serem de pouco interesse para um determinado grupo de usuários". Daí a importância da participação do especialista na coleta dos termos, seja apresentando seus assuntos de interesse ou esquemas de organização da informação, seja por meio da análise de registros de buscas já realizadas.

É preciso haver um equilíbrio entre esses dois princípios, pois é fundamental o atendimento às necessidades dos usuários. 
Dutra e Busch (2003) apontam a necessidade de recolher exemplares da documentação produzida e de vocabulários controlados existentes na organização, a fim de direcionar o desenvolvimento dessa etapa. Como procedimento de garantia de uso, ressaltamos o proposto por Yamaoka (2005), denominado "Rito para alteração ou inclusão de novos termos'. Por meio dele, os termos são sugeridos com base em um parecer, que é avaliado pela equipe de construção da taxonomia.

\section{Análise dos termos selecionados}

Após a coleta dos termos, é necessário padronizá-los, para que todos os usuários 'falem a mesma língua'. Desse modo, são estabelecidas algumas diretrizes, baseadas em Cavalcanti (1978).

a) singular e plural: primeiramente, verifica-se como o termo é utilizado no ambiente e se o significado muda no caso de uso do plural ou singular, como, por exemplo, Memória = faculdade de reter as idéias; e Memórias = registros de alguém que conta sua vida. Em geral, há a indicação do uso do singular nos seguintes casos: materiais (areia, cimento); propriedades específicas (condutividade); processo (seleção); nomes próprios (UNESCO); e disciplinas (matemática, gestão de pessoas). Nos casos de termos genéricos que designam classes de objetos, relações humanas, móveis, contratos de trabalho, orienta-se o uso do plural;

b) abreviaturas e siglas: usar somente quando facilmente reconhecidas ou quando o conceito é mais conhecido pela sigla, como por exemplo, PIB, ICMS, CPMF. Nesses casos, sempre construir remissivas para o nome em extenso, mantendo a consistência;

c) empréstimos linguísticos: a tradução para o português de termos em outras línguas dependerá da incorporação que esse termo sofreu na língua de especialidade, como, por exemplo, site ou sítio, e-mail ou correio eletrônico, call center ou central de atendimento. Alguns termos, em outras línguas, não apresentam correspondentes na língua portuguesa e são utilizados na forma original, como royalty, habeas corpus, software;

d) nomes populares e nomes científicos: a escolha de um ou de outro dependerá da área de atuação da organização e da preferência dos usuários; e

e) gíria ou jargão: dependerá das preferências linguísticas dos usuários, refletindo a manifestação lingüística mais frequente no ambiente.

Excluindo as questões relativas ao singular ou plural, deve-se utilizar a relação de equivalência para o controle das denominações de natureza sinonímica. 


\section{Controle da diversidade de significação}

Em ambientes em que o objetivo seja o compartilhamento de uma mesma linguagem, é necessário neutralizar a ambiguidade, entendida por Cintra et al. (2002, p. 21) "[...] como a possibilidade de uma comunicação lingüística prestar-se a mais de uma interpretação". A ambiguidade se impõe por meio da polissemia e da homonímia (CINTRA et al., 2002). A polissemia diz respeito à diversidade de significação de um mesmo termo; a homonímia refere-se a palavras com identidade de sons ou de forma, mas com significados distintos. Essas ambiguidades podem ser controladas por meio da contextualização do significado do termo. Essa contextualização é registrada entre parênteses, como ilustrado nos exemplos a seguir:

a) companhia (empresa); companhia (pessoa); e

b) capital (dinheiro); capital (sede do país).

Para neutralizá-las, pode-se, também, se necessário, elaborar "[...] redes relacionais e [estabelecer] definições e notas de escopo, sempre que as redes se mostrarem insuficientes para a interpretação unívoca da significação" (CINTRA et al., 2002, p. 33). Cavalcanti (1978) cita como exemplo o termo composto 'Organização da fábrica' que, para o sentido ser entendido, é necessário instituir uma nota explicativa (NE), como exemplificado abaixo:

$\mathrm{NE}$ : esquema de trabalho de uma fábrica

A elaboração das redes relacionais, auxiliares no controle da polissemia e da homonímia, será detalhada na próxima etapa.

Apesar de algumas das práticas de elaboração analisadas, nesta pesquisa, apontarem a problemática da ambiguidade, eles não aprofundam esta questão; portanto, não apresentam contribuição que possa ser utilizada como recomendação.

\section{Construção dos relacionamentos semânticos}

Nesta etapa, leva-se em consideração as relações semânticas entre os termos, que podem ser de equivalência, hierárquicas e nãohierárquicas, assim como polihierarquias.

As relações de equivalência se estabelecem entre termos sinônimos ou quase-sinônimos. A análise dos termos sinônimos precisa corresponder às necessidades e preferências do grupo de usuário. Seu controle deve ser realizado, permitindo a padronização e consequente aumento da qualidade da taxonomia. É importante que essa 'negociação' ocorra em parceria entre aquele que desenvolve a taxonomia e os usuários do sistema. Recomenda-se a utilização dos símbolos USE e UP (usado para). O USE tem a função de indicar o termo preferencial, enquanto que o UP tem o papel de indicar os termos proibidos, ou seja, aqueles que não podem ser utilizados para representar um assunto. A seguir, ilustra-se a utilização desses símbolos para o controle dos sinônimos

EMPRESA e ORGANIZAÇÃO. 


\author{
EMPRESA \\ USE ORGANIZAÇÃO \\ ORGANIZAÇÃO \\ UP EMPRESA
}

A taxonomia organiza a informação da mais genérica a mais específica, utilizando-se da relação gênero-espécie entre os termos. Esse tipo de relação é definido por Dahlberg (1978), como sendo o que aparece "entre dois conceitos que têm idênticas características, sendo, porém, que de uma relação à outra é apresentada uma característica adicional, de modo que surge entre eles uma hierarquia".

As relações hierárquicas podem ser do tipo gênero/espécie.

Relação gênero/espécie: exprime os graus de superordenação e subordinação entre os conceitos. O termo superordenado representa o termo genérico (TG), enquanto o termo subordinado representa o termo específico (TE).

\title{
MOVIMENTAÇÃO FINANCEIRA \\ TE APLICAÇÃO \\ APLICAÇÃO \\ TG MOVIMENTAÇÃO FINANCEIRA
}

O termo específico representa um conceito subordinado, que possui as mesmas características do conceito geral e mais alguma que o diferencia de outro conceito específico, subordinado ao mesmo conceito geral. Ele é considerado um tipo do termo genérico. No exemplo citado, APLICAÇÃO é um tipo de MOVIMENTAÇÃO FINANCEIRA.

A polihierarquia também deve ser considerada no estabelecimento das relações semânticas. Nela, um mesmo termo específico pode estar subordinado a mais de um termo genérico. Dutra e Busch (2003) demonstram preocupação com a representação dessa situação de significação. As relações de polihierarquia são frequentes no ambiente organizacional. O termo 'matéria-prima' é um exemplo, na área financeira, este conceito é considerado como um item de custo; na área de produção é um tipo de insumo. O termo 'matéria-prima' aparecerá ligado a dois termos genéricos, o que define a sua relação são as ligações semânticas estabelecidas na estrutura conceitual na qual está inserido.

As relações não-hierárquicas ou sequenciais (ou associativas) são muito frequentes em ambientes organizacionais, por representarem uma diversidade muito maior de relacionamentos semânticos. De acordo com Cintra et al. (2002), relações não-hierárquicas ou sequenciais são as que apresentam entre si contiguidade espacial ou temporal. No ambiente organizacional, as relações sequenciais que aparecem com maior frequência são:

a) relação de atribuição: 


\section{MARKETING DIVULGAÇÃO DE PRODUTOS}

b) processo ou operação/seu agente ou instrumento: PROGRAMAÇÃO SOFTWARE

c) matéria-prima/produto:
ALUMÍNIO
GRAMPO

d) ação/resultado da ação:

PLANEJAMENTO

e) efeito/causa:

EXPORTAÇÃO

f) atividades complementares:

COMPRA

g) ação/seu paciente:

VENDA

h) atividade/produto:

FUNDIÇÃO

i) associação implícita:

CRESCIMENTO ECONÔMICO
SOFTWARE

FERRO

PLANO DIRETOR

CRESCIMENTO ECONÔMICO

VENDA

\section{EXPORTAÇÃO}

Relação todo/parte: A norma ISO 2788 classifica a relação partitiva como hierárquica, nos casos de: 1) órgão do corpo humano: coração e sistema circulatório; 2) áreas geográficas: Brasil e São Paulo; e 3) disciplina: biologia e biologia vegetal. Nos demais casos, ela é tratada como não-hierárquica, pois, diferentemente da relação hierárquica gênero-espécie, na partitiva, o termo superordenado (um objeto ou conceito) é considerado um todo, do qual o termo subordinado é uma parte.

Utiliza os símbolos TE e TG e nos casos particulares citados pela norma ISO 2788, Termo geral partitivo e termo específico partitivo (TGP e TEP). Exemplo:

COMPUTADOR

TE HARDWARE

HARDWARE

TG COMPUTADOR
BRASIL

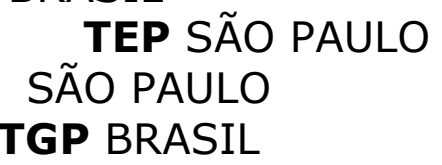

Lancaster (1987, p.26) acrescenta que:

[...] certas relações partitivas são freqüentemente tratadas como se fossem genéricas. Partes do corpo e nomes geográficos são os melhores exemplos. Faz sentido fazer de 'aorta' um TE subordinado a 'coração', embora ela seja uma 
parte e não um tipo de coração, o mesmo valendo para localização geográfica.

O mesmo acontece em relação a campos de estudo e estruturas sociais que são relações partitivas tratadas como genéricas.

As relações sequenciais, frequentemente expressas pela sigla TR (termo relacionado), são fundamentais para abranger a variedade de relações semântica passíveis de serem construídas dentro de uma organização. Sendo assim, no desenvolvimento de uma taxonomia, elas precisam ser contempladas a fim de garantir a qualidade da representação.

\section{Aplicação da taxonomia}

Em relação à aplicação da taxonomia na representação da informação (classificação ou indexação), o desenvolvimento das etapas anteriores influencia diretamente e, em consequência, afeta significativamente a recuperação da informação. Termos coletados e analisados, assim como relações semânticas estabelecidas, possibilitarão ou não a indexação adequada. Uma taxonomia bem construída facilitará atingir princípios e critérios de tratamento da informação determinados pelo portal corporativo. Por exemplo, uma taxonomia que representa os conceitos no nível de especificidade ${ }^{1}$ necessário, viabilizará a representação específica dos assuntos dos documentos. Esse fato provavelmente aumentará o nível de precisão ${ }^{2}$ na recuperação da informação. Por outro lado, a adoção do relacionamento não-hierárquico auxiliará a adoção do princípio de exaustividade, ou seja, a representação de todos os assuntos presentes em um documento, podendo colaborar com os níveis de revocação ${ }^{3}$ na recuperação da informação. Além disso, quando é utilizada uma taxonomia, os documentos têm maior probabilidade de serem indexados da mesma forma, atingindo, assim, a uniformidade no tratamento da informação e de sua recuperação.

Observando os princípios de especificidade e exaustividade, em relação aos critérios de revocação e precisão de um sistema de recuperação da informação, percebe-se que quanto maior o grau de especificidade, maior será a precisão e menor a revocação na recuperação. Na medida em que se diminui a especificidade, menor será a precisão, havendo uma probabilidade de aumento da revocação. A adoção do princípio de exaustividade atua de forma oposta. Quanto maior a exaustividade, maior a revocação e menor a precisão; quanto menor a exaustividade, menor a precisão e menor a revocação.

Sendo a opção pela especificidade e pela exaustividade diretamente dependente da correta construção de uma taxonomia, podemos deduzir

\footnotetext{
1 "Exatidão com que os descritores utilizados representam o conteúdo temático dos documentos" (PIEDADE, 1977, p. 4).

2 "Relação entre os documentos relevantes recuperados e o número total de documentos recuperados" (PIEDADE, 1977, p. 3).

3 "Relação entre os documentos relevantes recuperados e o número total de documentos relevantes sabidamente existentes na coleção" (PIEDADE, 1977, p. 3).
} 
que a qualidade da taxonomia influencia a qualidade da recuperação da informação.

Em relação à organização da informação recuperada do acervo, a taxonomia proporciona a categorização dos resultados de uma busca, facilitando a identificação das relações entre os assuntos. O mecanismo de busca do portal deve apresentar a saída dos resultados organizados nas categorias previamente definidas. Dessa forma, o usuário perceberá outros assuntos relacionados ao tema procurado que, muitas vezes, não Ihe haviam ocorrido. As categorias gerais estabelecidas na etapa ' $A$ ' para a organização dos termos são as mesmas utilizadas no momento da recuperação. É necessária a conexão entre a documentação do portal e as categorias da taxonomia no momento da aplicação, para que a taxonomia auxilie no processo de recuperação, com link direto aos recursos informacionais.

Conforme salienta Dutra e Busch (2003), a taxonomia precisa servir de referência para o entendimento da área do conhecimento que se dispõe a representar.

\section{Considerações finais}

Este trabalho buscou propor princípios básicos para o processo de construção de taxonomias em portais corporativos, no sentido de apresentar uma discussão teórica mais aprofundada acerca de suas etapas. A proposição das etapas de construção da taxonomia partiu da análise de práticas de elaboração apresentadas na literatura da área de Ciência da Informação.

Apesar dos portais corporativos indicarem as taxonomias como elementos centrais no processo de representação e organização da informação, a literatura da área não apresenta indicações metodológicas claras, em relação ao seu desenvolvimento. Esse problema ocorre principalmente com a etapa da construção das relações semânticas entre os termos. Podemos constatar que a taxonomia trabalha os conceitos básicos da classificação, aplicados em um ambiente diferenciado - a Internet. Desse modo, para a construção de taxonomias, o processo precisa estar respaldado com as teorias e métodos elaborados pelas áreas de Classificação, Indexação, Lingüística e Documentação. Essas teorias e métodos fornecem o devido aprofundamento teórico e metodológico necessário nas etapas da construção.

\section{Referências}

CAMPOS, M. L. A. Linguagem documentária: teorias que fundamentam sua elaboração. Niterói: EdUFF, 2001.

CAVALCANTI, C. R. Indexação e tesauro: metodologia e técnicas. Brasília: Associação dos bibliotecários do Distrito Federal, 1978.

CINTRA, A. M. M. et al. Para entender as linguagens documentárias. São Paulo: Polis, 2002. 
CLASSIFICATION RESEARCH GROUP. The need for a faceted classification as the basis of all methods of information retrieval. Library Association Record, London, v. 57, n. 7, p. 262-268, 1955.

DAHLBERG, I. Fundamentos teórico-conceituais da classificação. Revista de Biblioteconomia de Brasília, v. 6, n. 1, p. 9-21, jan./jun. 1978.

DUTRA, J.; BUSCH, J. Enabling knowledge discovery: taxonomy development for NASA. 2003. Disponível em: < http://www.xml.gov/documents/completed/nasa/index.html>. Acesso em: 04 out. 2006.

EARLEY AND ASSOCIATES. Developing enterprise taxonomies. 2003. Disponível em: <http://www.earley.com/Earley Report/ER Taxonomy.htm>. Acesso em: 04 out. 2006.

KREMER, S.; KOLBE, L. M.; BRENNER, W. Towards a procedure model in terminology management. Journal of documentation, Bradford:UK, v. 61, n. 2, p. 281-295, 2005.

LANCASTER, F. W. Construção e uso de tesauro: curso condensado. Brasília: IBICT, 1987.

LANGRIDGE, D. Classificação: abordagem para estudantes de biblioteconomia. Rio de Janeiro: Interciência, 1977.

MARTINEZ, A. et al. Las categorías o facetas fundamentales: uma metodología para el diseño de taxonomías corporativas de sitios Web argentinos. Ci. Inf., Brasília, v. 33, n. 2, p. 106-111, maio/ago. 2004.

MONTAGUE INSTITUTE. Managing taxonomies strategically. 2001. Disponível em: <http://www.montague.com/abstracts/taxonomy3.html>. Acesso em: 26 out. 2006.

MORANTE, M. Creating useful taxonomies: metadata, taxonomies and controlled vocabularies. 2003. Disponivel em: <htt://www.kcurve.com/Metadata_Taxonomy\%20Development_SLA_060 804.ppt>. Acesso em: 03 nov. 2006.

PIEDADE, M. A. R. Introdução à teoria da classificação. Rio de Janeiro: Interciência, 1977.

RAMSDEN, M. J. An introduction to index language construction, a programed text. London: C. Bingley, 1974 apud PIEDADE, M. A. R. Introdução à teoria da classificação. Rio de Janeiro: Interciência, 1977.

RANGANATHAN, S.R. Facet analysis: fundamental categories. 1962. In.: CHAN, L. M. Theory of subject analysis. Colorado: Libraries Unlimited, 1985. p. 86-93.

TERRA, J. C. C.; GORDON, C. Portais corporativos: a revolução na gestão do conhecimento. São Paulo: Elsevier, 2002.

VICKERY, B. C. Faceted classification: a guide to the construction and use of special schemes. Londres: Aslib, 1960. 
YAMAOKA, E.J. Taxonomia e metadados como elementos estruturantes da organização e representação do conhecimento numa empresa. 2005. 151f. Dissertação (Mestrado em Ciência da Informação) - Universidade de Brasília, Brasília, 2005. 